\title{
Conchostráceos com Linhas de Crescimento Recurvadas Junto à Margem Dorsal (Famílias Palaeolimnadiopseidae e Perilimnadiidae) da Formação Rio do Rasto, Permiano Superior, Bacia do Paraná, Brasil
}

\author{
Luis Gustavo Ferreira-Oliveira (lgfo@rc.unesp.br) e Rosemarie Rohn (rohn@rc.unesp.br) \\ Departamento de Geologia Aplicada - Instituto de Geociências e Ciências Exatas - UNESP \\ Av. 24-A 1515, CEP 13506-900, Rio Claro, SP, BR
}

Recebido em 27 de junho de 2007; aceito em 07 de fevereiro de 2008

Palavras-chave: Conchostraca, Palaeolimnadiopseidae, Perilimnadiidae, bacia do Paraná, Permiano superior.

\section{RESUMO}

\begin{abstract}
Alguns conchostráceos permianos da Formação Rio do Rasto (Bacia do Paraná, Brasil) apresentam um encurvamento bem característico nas linhas de crescimento na região próxima à margem dorsal. Todos os exemplares previamente descritos foram atribuídos a Palaeolimnadiopsis subalata (Reed) Raymond. Porém, uma reanálise deste material fóssil e de exemplares adicionais demonstrou que nem todos os espécimes podem ser incluídos em um mesmo táxon, nem em uma mesma família (Palaeolimnadiopseidae). De acordo com a forma da carapaça e tamanho do umbo, estes exemplares são aqui referidos a três espécies. As carapaças de formato subelíptico, com umbo pequeno e em posição subanterior são mantidas em Palaeolimnadiopsis subalata (Reed, 1929) Raymond, 1946. As carapaças subcirculares com umbo pequeno em posição subcentral correspondem a Palaeolimnadiopsis riorastensis sp. nov. As valvas de formato elíptico com umbo grande e anterior são atribuídas à Falsisca brasiliensis sp. nov., da Família Perilimnadiidae. O gênero Palaeolimnadiopsis tem uma ampla distribuição cronoestratigráfica, porém o gênero Falsisca está restrito ao Permiano superior - Triássico inferior, da Europa e da Ásia. Este intervalo está de acordo com a provável idade permiana superior dos estratos onde foram encontrados estes fósseis na Formação Rio do Rasto. O gênero Falsisca é aqui referido pela primeira vez ao registro fóssil do Gondwana.
\end{abstract}

Keywords: Conchostraca, Palaeolimnadiopseidae, Perilimnadiidae, Paraná Basin, Upper Permian.

\begin{abstract}
Some Upper Permian conchostracans from the Rio do Rasto Formation (Paraná Basin, South Brazil) have very characteristic recurved growth lines at the dorsal margin. All previously described specimens were classified as Palaeolimnadiopsis subalata (Reed) Raymond. However, a re-analysis of these fossils and of additional recently-collected specimens demonstrated that not all can be included in a single species, nor only in the Family Palaeolimnadiopseidae. According to their shape and the size of the umbo, they are classified into three species. The sub-elliptic carapaces with small anterior umbo are maintained in Palaeolimnadiopsis subalata (Reed, 1929) Raymond, 1946. The sub-circular carapaces with small sub-central umbo correspond to the new species Palaeolimnadiopsis riorastensis. The small size of the umbo is a character of the Family Palaeolimnadiopseidae. The small elliptic valves with large anterior umbo are assigned to the new species Falsisca brasiliensis of the Family Perilimnadiidae, which is characterized by large umbos. Palaeolimnadiopsis has a wide chronostratigraphic distribution, but Falsisca is restricted to the Upper Permian-Lower Triassic of Europe and Asia. This interval is in agreement with the probable Late Permian age of the respective strata of the Rio do Rasto Formation. Falsisca was not previously recorded in Gondwana.
\end{abstract}




\section{INTRODUÇÃO}

Conchostráceos são pequenos crustáceos que possuem carapaças bivalves quitinosas, cujas espécies viventes habitam tipicamente corpos de água doce. Apenas a porção superior da espessa sucessão permo-carbonífera da Bacia do Paraná (sul do Brasil), i.e., a Formação Rio do Rasto do Grupo Passa Dois, contém sedimentos de origem continental inequívoca e abundantes conchostráceos fósseis. Em pelo menos 192 afloramentos da formação há registros destes crustáceos (Mendes, 1954; Rohn, 1987, 1994; Tasch, 1987) e diversas espécies estão sendo revisadas quanto a sua classificação (e.g., 'Monoleaia' unicostata, 'Paranaleaia', 'Gabonestheria', 'Cyzicus', 'Asmussia', Palaeolimnadia).

O presente trabalho analisa conchostráceos da Formação Rio do Rasto com recurvamento das linhas de crescimento junto à margem póstero-dorsal. Tal característica tem sido usada (em Raymond, 1946; Mendes, 1954; Rohn, 1987, 1994) para classificá-los no gênero Palaeolimnadiopsis Raymond, 1946 e na Família Palaeolimnadiopseidae Defretin, 1965. Porém, outras famílias também podem apresentar o recurvamento das linhas de crescimento, fato que é discutido neste trabalho.

\section{MATERIAIS E MÉTODOS}

O material aqui estudado compreende exemplares já descritos por Reed (1929), Mendes (1954) e Rohn (1987, 1994), além de alguns exemplares coletados mais recentemente. Consiste ele de moldes de 16 valvas, pertencentes às coleções paleontológicas das seguintes instituições: Universidade Estadual Paulista - Campus de Rio Claro - SP (UNESP), sigla URC AC; Universidade de São Paulo (USP), sigla GP1T; e Departamento Nacional da Produção Mineral - Rio de Janeiro (DNPM), com a sigla DNPM.

Os exemplares URC AC 161 foram coletados em Três Bicos, a 35,4 km de Cândido de Abreu e a 41,5 km da cidade de Reserva (Afloramento 1, Figura 1), Estado do Paraná, em uma fazenda situada às margens da rodovia PR 239, no km 81,5(UTM 22J 490,22/7272,78).

O exemplar URC AC 175 é procedente de um afloramento às margens da rodovia PR 487, a 14,2 km de Cândido de Abreu (Afloramento 2, Figura 1), Estado do Paraná (UTM 22J 376,04/7273,98).

O exemplar GP1T 1609A foi coletado também às margens da rodovia PR 239, no município de Três Bicos, a 37,5 km de Cândido de Abreu e 39,4 km de Reserva (Afloramento 3, Figura 1).

Os exemplares DNPM 1420, GP1T 1509 A e GP1T 1504 provêm do município de Poço Preto, Estado de Santa Catarina, encontrado "em folhelhos vermelhos do Rio
Iguassú, na povoação de Vallões” (sic) (Reed, 1929) que atualmente corresponde à rodovia BR 280, a $200 \mathrm{~m}$ a leste do Rio Timbó, próximo à confluência com o rio Iguaçu (Afloramento 4, Figura 1).

Os exemplares foram analisados e fotografados com o auxílio de uma lupa estereoscópica com câmera digital acoplada, além de imagens obtidas em um microscópio eletrônico de varredura (MEV), para observação mais acurada de detalhes morfológicos e ornamentação.

\section{GEOLOGIA E LOCALIZAÇÃO DOS AFLORAMENTOS}

A Formação Rio do Rasto é a unidade superior do Grupo Passa Dois na faixa aflorante no Sul do Brasil e é subdividida nos membros Serrinha e Morro Pelado. O Membro Morro Pelado, com $250 \mathrm{~m}$ de espessura, é caracterizado por lamitos vermelhos, ritmitos e arenitos de granulometria relativamente fina. Vários arenitos lobados são interpretados como barras de desembocadura de rios efêmeros em lagos com pouca profundidade ou depósitos de rompimento de diques marginais. Fácies típicas de canais fluviais são raras (Rohn, 1994). Arenitos de dunas eólicas aumentam em direção ao topo do Membro Morro Pelado. O registro fóssil é representado principalmente por vegetais pouco diversificados, raros moluscos bivalves, restos de peixes, raros tetrápodos, bem como conchostráceos relativamente abundantes e diversificados (Rohn, 1994). Próximo ao topo da formação, onde depósitos de inundação e/ou lacustres efêmeros se alternam com depósitos de dunas eólicas, ocorre um intervalo relativamente pequeno (aproximadamente $40 \mathrm{~m}$ ) com maior diversidade de conchostráceos, destacando-se os exemplares analisados neste trabalho.

\section{SISTEMÁTICA PALEONTOLÓGICA}

Ordem Conchostraca Sars, 1867.

Subordem Spinicaudata Linder, 1945.

Superfamília Lioestheriodea Raymond, 1946.

Família Paleolimnadiopseidae Defretin-Le Franc, 1965.

Subfamília Paleolimnadiopseinae Defretin-Le Franc, 1965.

Gênero Palaeolimnadiopsis Raymond, 1946.

Espécie Tipo Palaeolimnadiopsis carpenteri Raymond, 1946.

Diagnose: Paleolimnadiopseidae com bandas de crescimento apresentando uma ornamentação reticulada fina ou quase ausente e linhas de crescimento sem nódulos ou espinhos (Shen, 1985).

Cronoestratigrafia: Devoniano Médio a Cretáceo Superior (Shen, 1985).

Distribuição geográfica: Europa, Ásia, América do Sul e do Norte. 

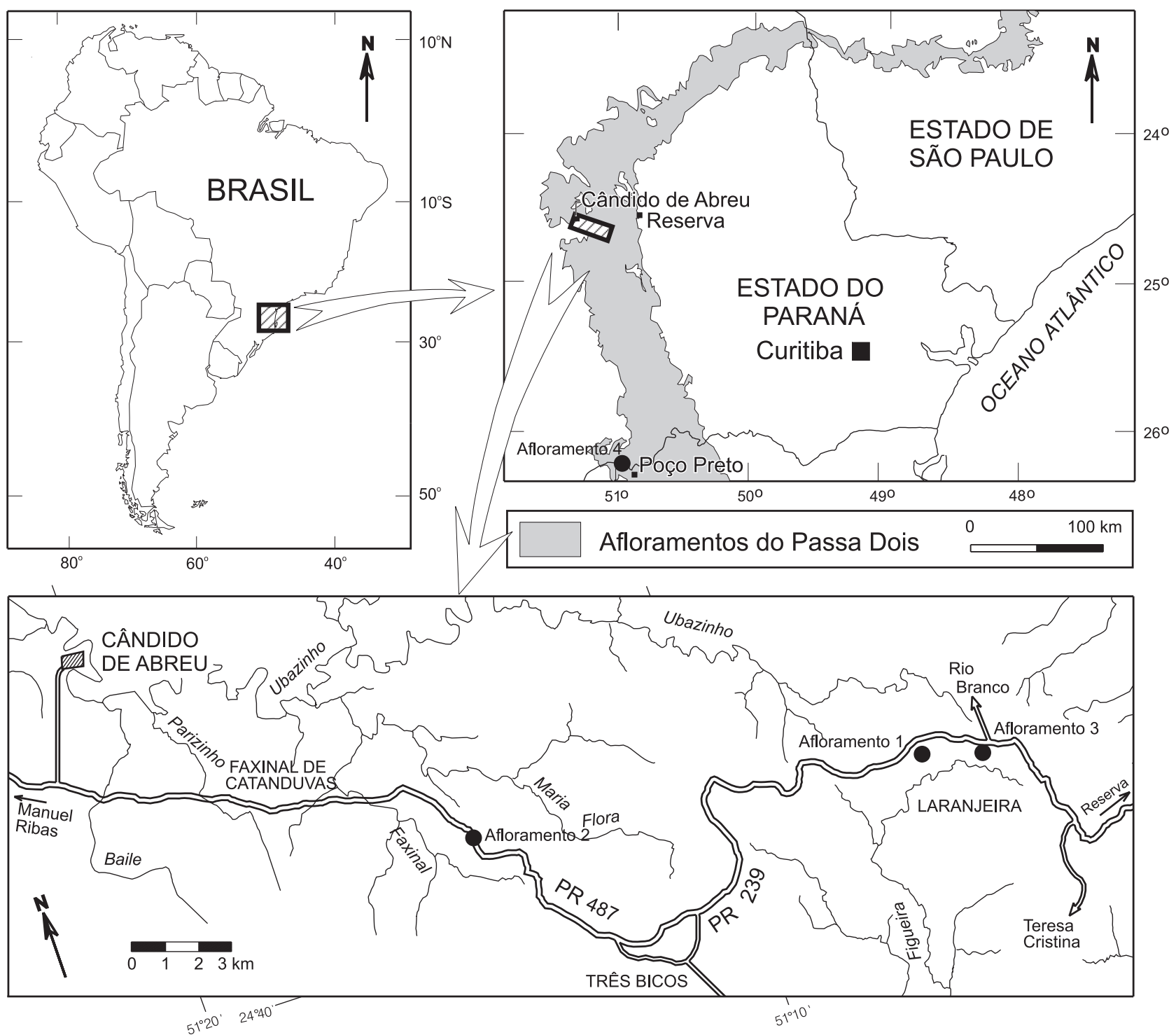

\section{Afloramentos}

Rios

Rodovias

Figura 1. Localização dos afloramentos estudados.

\section{Palaeolimnadiopsis subalata (Reed, 1929) Raymond, 1946}

(Figuras 2 e 3 )

Sinonímias: Estheria subalata Reed, 1929; Palaeolimnadiopsis subulata (sic) (Reed, 1929) Raymond, 1946.

Holótipo: DNPM 1420 A.

Localidade-tipo e estratigrafia: município de Poço Preto, Estado de Santa Catarina, Brasil, rodovia BR 280, a $200 \mathrm{~m}$ a leste do Rio Timbó, próximo à confluência com o Rio Iguaçu, porção superior do Membro Morro Pelado, Formação Rio do Rasto.

Outros exemplares estudados, localização e estratigrafia

URC AC 161 A, B, C e Ga. Localização: “Afloramento 1” em Três Bicos, a 35,4 km de Cândido de Abreu e a 41,5 km da cidade de Reserva, Estado do Paraná, em uma fazenda situada às margens da rodovia PR 239, no km 81,5 (UTM 

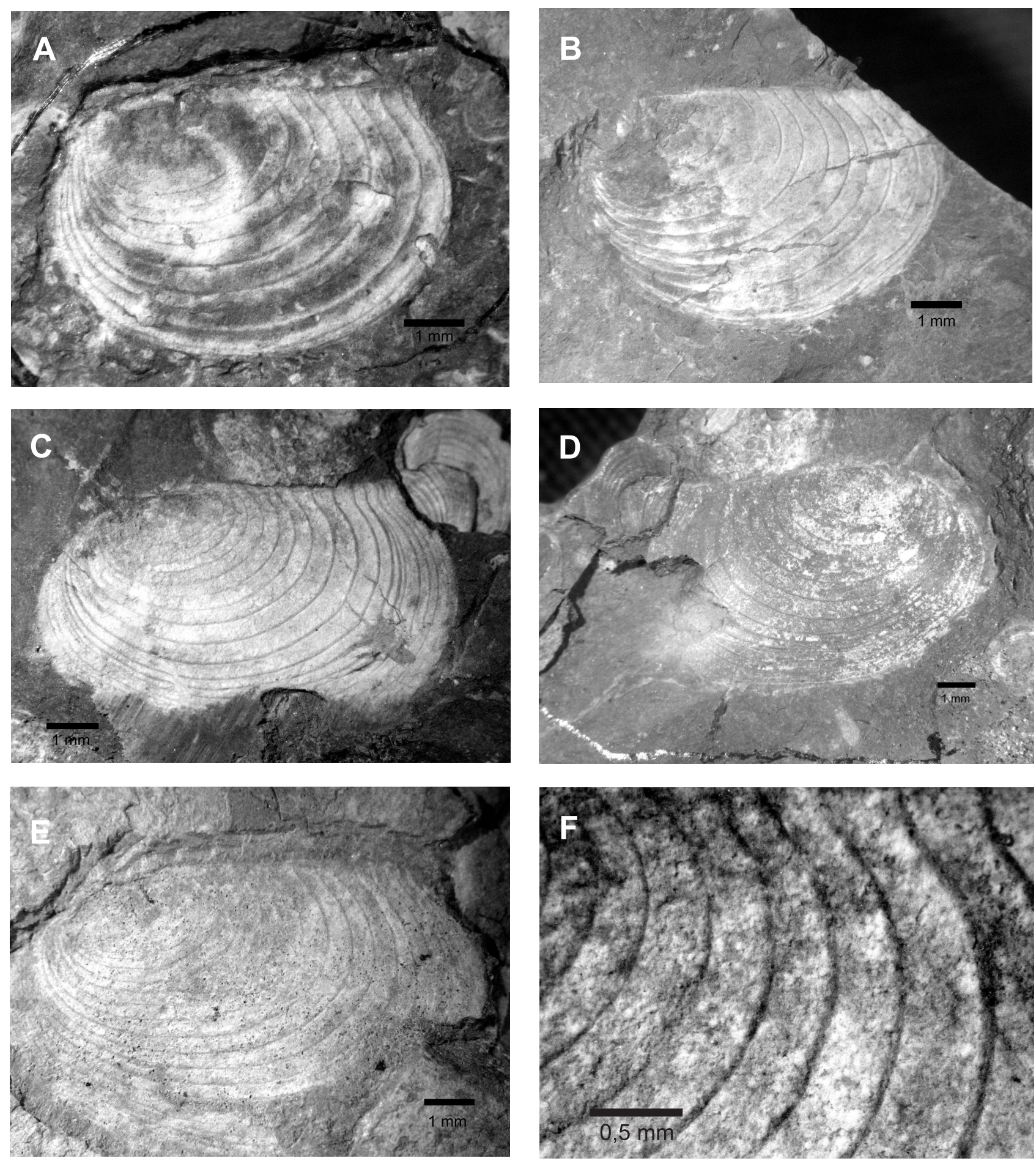

Figura 2. Palaeolimnadiopsis subalata. A. Exemplar URC AC 161 A. B. Exemplar URC AC 161 B. C. Exemplar URC AC 161 C. D. Exemplar URC AC $161 \mathrm{Ga}$. E. Exemplar URC AC 175 A. F. Exemplar URC AC 161 C (detalhe da ornamentação). 


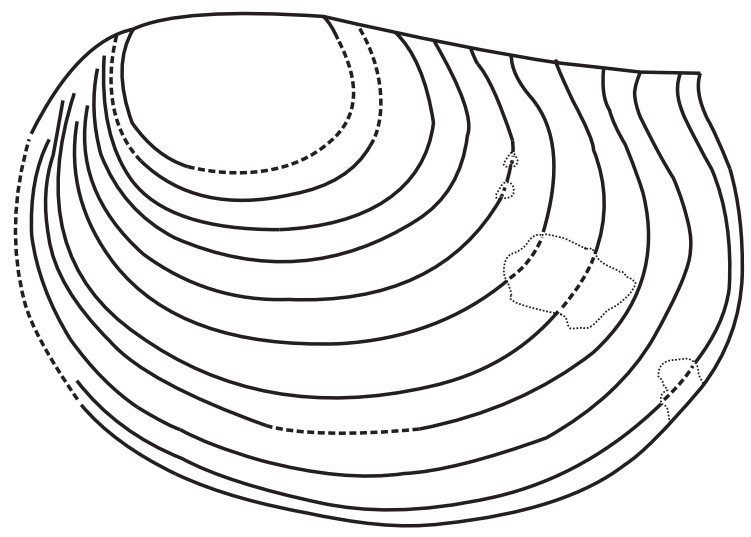

$1 \mathrm{~mm}$

Figura 3. Esquema da morfologia externa de Palaeolimnadiopsis subalata.

22J 490,22/7272,78), porção superior do Membro Morro Pelado, Formação Rio do Rasto.

URC AC 175. Localização: “Afloramento 2”, às margens da rodovia PR 487, a 14,2 km de Cândido de Abreu, no Estado do Paraná (UTM 22J 376,04/7273,98), também na porção superior do Membro Morro Pelado, Formação Rio do Rasto.

Diagnose emendada: carapaça de tamanho médio e de forma subelíptica. Umbo de tamanho pequeno, anterior. Margem dorsal reta, margem ventral fortemente arqueada. Linhas de crescimento numerosas, em número de 10 a 21. Linhas de crescimento mais externas, abrangendo cerca da metade do total, caracterizadas por uma curvatura ligeiramente côncava na porção posterior próxima à margem dorsal. Bandas de crescimento largas na região centro-posterior da valva e geralmente mais estreitas na região próxima à margem ventral. Ornamentação poligonal muito tênue nas bandas de crescimento.
Emended diagnosis: subelliptic mid-sized carapace. Small anterior umbo. Straight dorsal margin, strong arched ventral margin. Many growth lines, from 10 to 21 . External growth lines, about half of all, characterized by a slightly concave shape at the posterior end next to the dorsal margin. Growth bands broad at the mid-posterior region of the valve and generally narrower next to the ventral margin. Very faint polygonal ornamentation on the growth bands.

Descrição: os exemplares analisados correspondem a valvas de tamanho médio, com contorno elíptico, comprimento da valva variando entre 6,0 e $12 \mathrm{~mm}$ e altura entre 4,0 e 8,0 mm; proporção entre a altura máxima e o comprimento igual a 0,66. A margem dorsal é reta. A margem ventral é fortemente arqueada. Apresenta linhas de crescimento em número de 10 a 21, recurvadas na margem posterior próximo à margem dorsal da valva, geralmente a partir de um terço do total das linhas de crescimento. O umbo é pequeno e anterior. O espaçamento entre as linhas de crescimento é relativamente uniforme - até $0,5 \mathrm{~mm}$ na região póstero-ventral e menor nas regiões anterior e ventral. A ornamentação está pouco preservada, porém, nos exemplares onde pode ser observada, é constituída por pequenos polígonos com diâmetros entre 0,1 e 0,2 mm. Os dados quantitativos dos espécimes encontram-se na Tabela 1.

Discussão: entre os exemplares originalmente atribuídos a $P$. subalata por Reed (1929), um apresenta umbo pequeno e subanterior (Reed, 1929: est. 1, figura 9) e dois possuem umbo grande e anterior (Reed, 1929: est. 1, figuras 10 e 11). Somente o exemplar da figura 9 em Reed (1929) foi mantido na espécie, pois o tamanho do umbo tem forte relação com o desenvolvimento ontogenético dos conchostráceos, sendo característica diagnóstica fundamental inclusive na classificação de famílias (vide abordagem detalhada ao final do trabalho). Shen (1985), Chen e Shen (1985) e Novojilov (1970) discriminaram Paleolimnadiopseidae de outras famílias com a margem posterior recurvada em vista do tamanho do umbo. Por esta razão, tornou-se necessário modificar a diagnose de $P$. subalata, no sentido de abranger apenas exemplares com umbo pequeno. Quase todas as demais características observadas inicialmente por Reed

Tabela 1. Dados morfométricos de Palaeolimnadiopsis subalata (Reed, 1929) Raymond, 1946.

\begin{tabular}{ccccc}
\hline Exemplar & Altura $(\mathbf{m m})$ & Comprimento $(\mathbf{m m})$ & H/L & 0,66 \\
URC AC 161 A & 4,0 & 6,0 & 0,66 & 10 \\
URC AC 161 B & 6,0 & 9,0 & 0,66 & 13 \\
URC AC 161 C & 6,0 & 9,0 & 0,66 & 19 \\
URC AC 161 Ga & 6,0 & 9,0 & 0,66 & 21 \\
URC AC 175 A & 8,0 & 12,0 & 0,66 & 19 \\
DNPM 1420 A & 5,3 & 8,0 & & 12 \\
\hline
\end{tabular}


(1929) foram mantidas na diagnose, exceto a ornamentação. Mendes (1954) já havia comentado que a ornamentação seria muito inconspícua. Entre os exemplares aqui estudados, raros apresentam alguma ornamentação, sendo constituída por pequenos polígonos mal preservados (Figura $2 \mathrm{~F}$ ).

\section{Palaeolimnadiopsis riorastensis sp. nov.}

(Figuras 4 e 5)

Sinonímia: cf. Palaeolimnadiopsis sp. em Rohn (1987). Holótipo: URC AC $161 \mathrm{E}$.

Localidade-tipo e estratigrafia: “Afloramento 1” em Três Bicos, a 35,4 km de Cândido de Abreu e a 41,5 km da cidade de Reserva, Estado do Paraná, em uma fazenda situada às margens da rodovia PR 239, no km 81,5 (UTM 22J 490,22/7272,78), porção superior do Membro Morro Pelado, Formação Rio do Rasto.
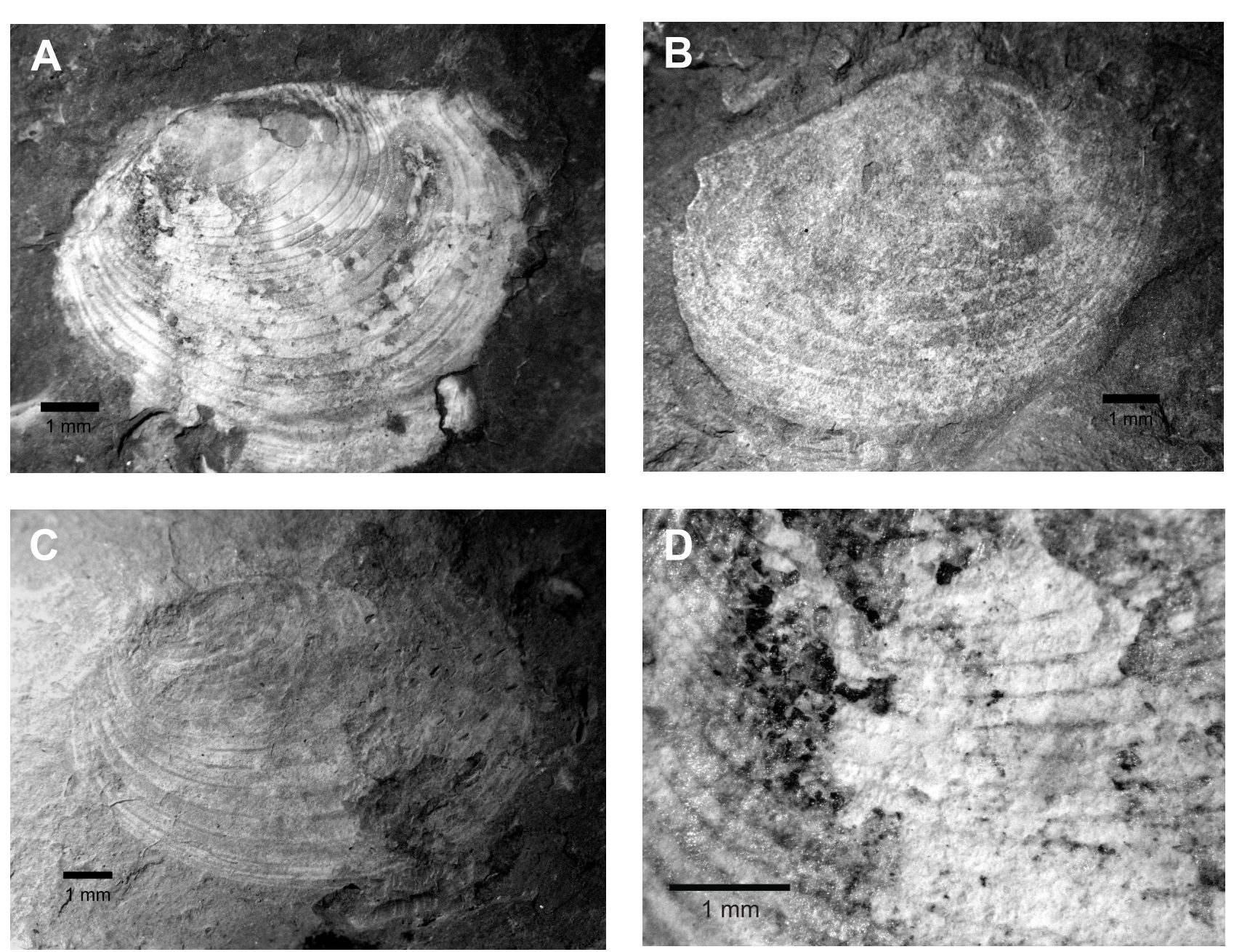

Figura 4. Palaeolimnadiopsis riorastensis sp. nov. A. Exemplar URC AC 161 E. B. Exemplar URC AC 175 B. C. Exemplar GP1T 1609 A. D. Exemplar URC AC 161 E (detalhe da ornamentação).

\section{Parátipos, procedência e estratigrafia}

URC AC 175 B. Localização: “Afloramento 2”, às margens da rodovia PR 487, a 14,2 km de Cândido de Abreu, no Estado do Paraná (UTM 22J 376,04/7273,98), porção superior do Membro Morro Pelado, Formação Rio do Rasto.

GP1T 1609 A. Localização: “Afloramento 3”, às margens da rodovia PR 239, no município de Três Bicos, a 37,5 km de Cândido de Abreu e 39,4 km de Reserva.

Epíteto específico: riorastensis, em alusão à Formação Rio do Rasto, onde o holótipo foi coletado.

Diagnose: carapaça de tamanho médio e de forma subcircular. Umbo de tamanho pequeno, subcentral. Margem dorsal levemente arqueada, margem ventral fortemente arredondada. Linhas de crescimento numerosas, em número de 19 a 25, apresentando uma curvatura ligeiramente côn- 


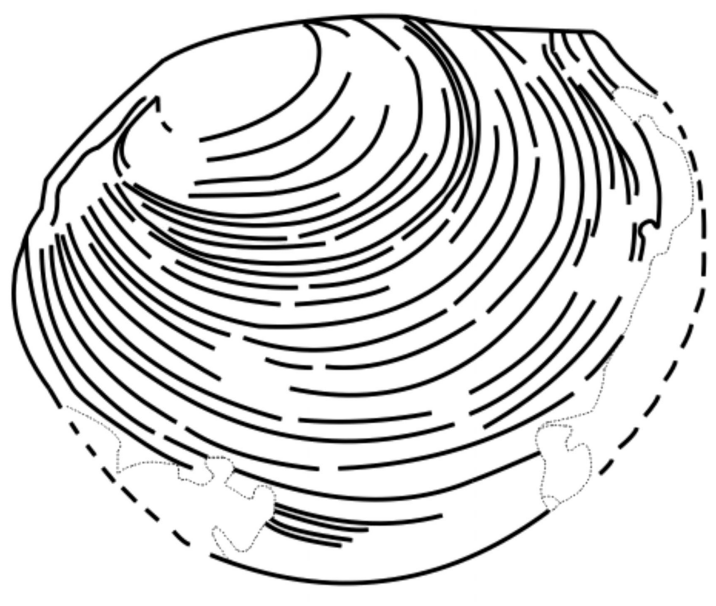

$1 \mathrm{~mm}$

Figura 5. Esquema da morfologia externa de Palaeolimnadiopsis riorastensis sp. nov.

cava na margem posterior próxima à margem dorsal, geralmente a partir da quinta linha de crescimento das valvas. Bandas de crescimento variáveis em largura. Ornamentação hachurada radialmente nas bandas de crescimento.

Diagnosis: subcircular mid-sized carapace. Small subcentral umbo. Slightly arched dorsal margin, strong rounded ventral margin. Many growth lines, from 19 to 25, characterized by a slightly concave shape at the posterior end next to the dorsal margin from about the fifth growth line on. Width of the growth bands variable. Radial hatched ornamentation on the growth bands.

Descrição: os exemplares analisados correspondem a valvas de tamanho médio, com contorno subcircular, comprimento da valva variando 9,0 e 10,5 mm e altura entre 6,5 e 7,5 mm; proporção entre a altura máxima e o comprimento entre 0,71 e 0,72 . O umbo é pequeno e subcentral. A margem póstero-dorsal é ligeiramente convexa próxima à região umbonal e levemente côncava próxima ao contato entre esta e a margem posterior. Seu comprimento corresponde aproximadamente ao dobro da margem ântero-dorsal. A margem ventral é fortemente arqueada. Os exemplares apresentam numerosas linhas de crescimento, entre 19 a 25 linhas, recurvadas na margem posterior próxima à margem dorsal da valva, geralmente a partir de um terço do total das linhas de crescimento ou aproximadamente a partir da quinta linha. O espaçamento entre as linhas de crescimento não é uniforme, variando nas regiões das valvas. A ornamentação está pouco preservada, porém, nos exemplares onde se pode observá-la, apresenta-se em forma de hachuras radiais paralelas como em Lioestheria (largura entre as hachuras = 0,15 a 0,20 mm). Os dados quantitativos dos espécimes encontram-se na Tabela 2.

Discussão: Rohn (1987) observou que raros exemplares identificados como cf. Palaeolimnadiopsis sp. da Formação Rio do Rasto, mal preservados na região póstero-dorsal e sem recurvamento claro das linhas de crescimento, seriam distintos daqueles tradicionalmente classificados como $P$. subalata, especialmente pelo umbo bem pequeno, número grande de linhas de crescimento e valvas de dimensões relativamente maiores e circulares. Após coleta de exemplares adicionais, agora melhor preservados, tais diferenças puderam ser confirmadas. $\mathrm{O}$ formato subcircular das valvas, com margem póstero-dorsal arqueada e o umbo em posição subcentral, dividindo a margem dorsal nas suas porções anterior e posterior na proporção de 1:2 são características exclusivas de $P$. riorastensis sp. nov. Esta espécie assemelhase a Endolimnadiopsis rusconii Gallego, 2005 do Triássico da Argentina, porém a margem dorsal é menos reta, o formato é mais subcircular do que oval e a ornamentação é bem mais tênue, embora também reticular. $P$. riorastensis sp. nov. distingue-se de quaisquer outros paleolimnadiopseídeos conhecidos, o que justifica a sua inclusão em uma nova espécie.

Ordem Conchostraca Sars, 1867.

Subordem Spinicaudata Linder, 1945.

Família Perilimnadiidae Chang e Chen, 1975.

Gênero Falsisca Novojilov 1970.

Genótipo: Falsisca turaica Novojilov, 1965.

Cronoestratigrafia: Permiano superior-Triássico inferior. Distribuição geográfica: Europa, Ásia e América do Sul.

Diagnose genérica: Valva elíptica, margem dorsal reta ou um pouco arqueada. Margem posterior próxima à margem dorsal ligeiramente côncava. Umbo grande, bandas de crescimento largas, linhas de crescimento em número de 4 a 22. Ornamentação finamente reticulada (ou pontuada) (Novojilov, 1970).

Tabela 2. Dados morfométricos de Palaeolimnadiopsis riorastensis sp. nov. NC = não consta este dado.

\begin{tabular}{ccccc}
\hline Exemplar & Altura $(\mathbf{m m})$ & Comprimento $(\mathbf{m m})$ & H/L & Linhas de crescimento \\
\hline URC AC 175 B & 6,7 & 9,0 & 0,71 & NC \\
GP1T 1609 A & 7,5 & 10,5 & 0,71 & 19 \\
URC AC 161 E & 6,5 & 9,0 & 0,72 & 25 \\
\hline
\end{tabular}




\section{Falsisca brasiliensis sp. nov.}

(Figuras 6 e 7 )

\section{Holótipo: DNPM 1420 B}

Localidade-tipo e estratigrafia: município de Poço Preto, Estado de Santa Catarina, Brasil, rodovia BR 280, a $200 \mathrm{~m}$ a leste do Rio Timbó, próximo à confluência com o Rio Iguaçu, porção superior do Membro Morro Pelado, Formação Rio do Rasto.

\section{Parátipos, procedência e estratigrafia}

URC AC 161 D e Gb. Localidade: “Afloramento 1” em Três Bicos, a 35,4 km de Cândido de Abreu e a 41,5 km da cidade de Reserva, Estado do Paraná, em uma fazenda situada às margens da rodovia PR 239, no km 81,5 (UTM 22J
490,22/7272,78), porção superior do Membro Morro Pelado, Formação Rio do Rasto.

GP1T 1509A e GP1T 1504. Localidade: município de Poço Preto, Estado de Santa Catarina, Brasil, rodovia BR 280, a $200 \mathrm{~m}$ a leste do Rio Timbó, próximo à confluência com o Rio Iguaçu, porção superior do Membro Morro Pelado, Formação Rio do Rasto (Afloramento 4).

DNPM 1420 C. Localidade: município de Poço Preto, Estado de Santa Catarina, Brasil, rodovia BR 280, a $200 \mathrm{~m}$ a leste do Rio Timbó, próximo à confluência com o Rio Iguaçu, porção superior do Membro Morro Pelado, Formação Rio do Rasto.

Epíteto específico: brasiliensis, por ser a única ocorrência do gênero Falsisca registrada no Brasil até o momento.
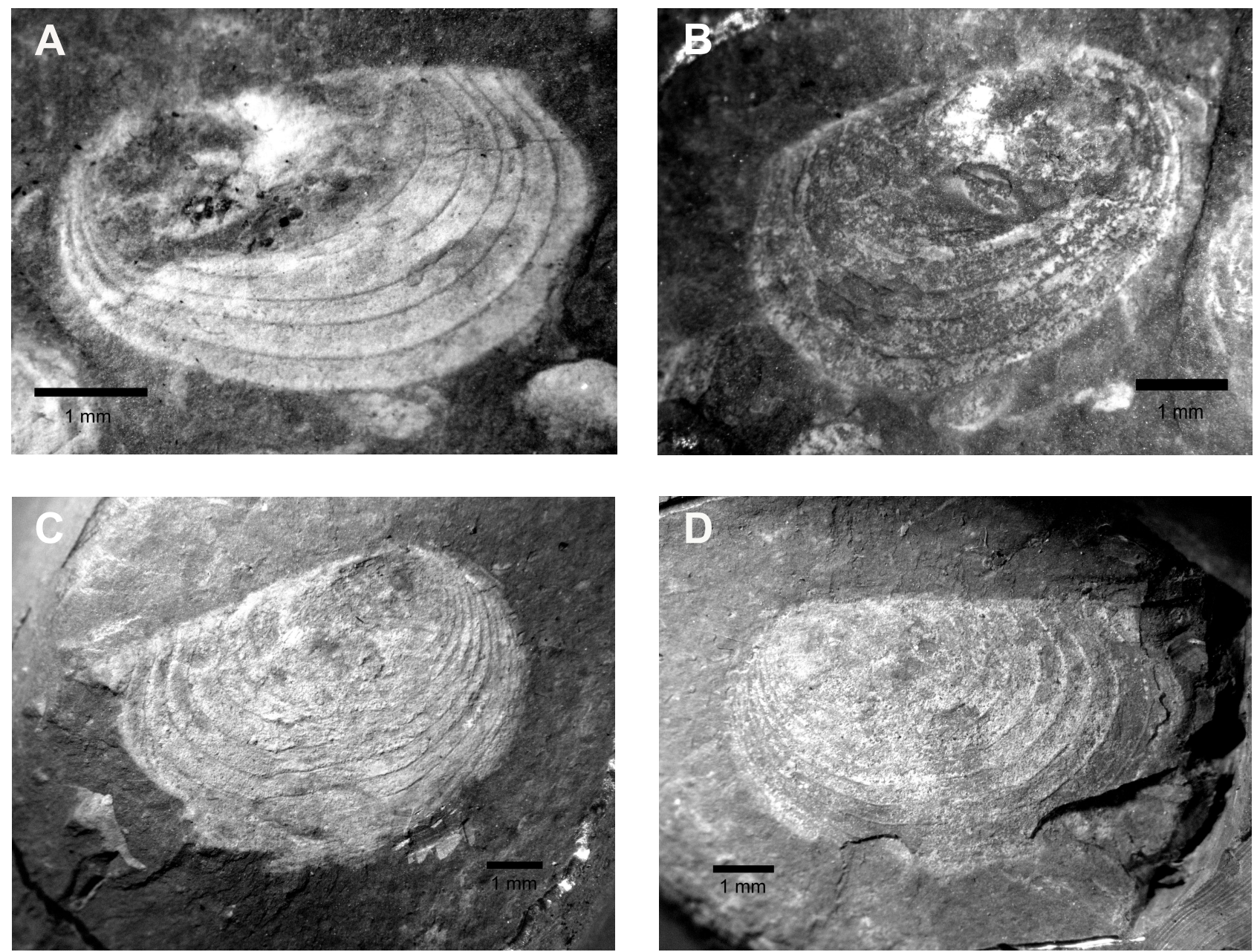

Figura 6. Falsisca brasiliensis sp. nov. A. Exemplar URC AC 161 D. B. Exemplar URC AC 161 Gb. C. Exemplar GP1T 1509. D. Exemplar GPIT 1504. 


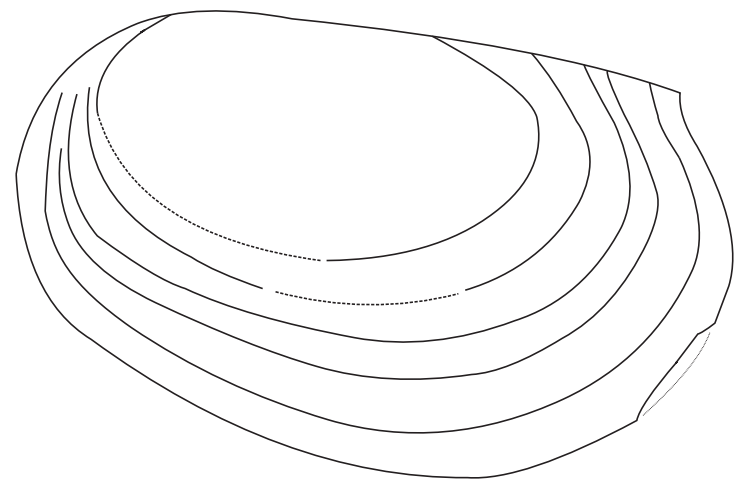

$1 \mathrm{~mm}$

Figura 7. Esquema da morfologia externa de Falsisca brasiliensis sp. nov.

Diagnose: Valvas pequenas de formato elíptico. Umbo grande, anterior. Margem dorsal reta ou levemente arqueada. Margem ventral arqueada. Linhas de crescimento largamente espaçadas em número de 7 a 11 . Linhas de crescimento mais externas, abrangendo cerca da metade do total, caracterizadas por uma curvatura ligeiramente côncava na porção posterior próxima à margem dorsal.

Diagnosis: Small elliptic valves. Large anterior umbo. Straight or slightly arched dorsal margin. Arched ventral margin. Widely spaced growth lines, 7 to 11 in number. External growth lines, about half of all, characterized by a slightly concave shape at the posterior end next to the dorsal margin.

Descrição: Os exemplares analisados correspondem a valvas de tamanho pequeno, com contorno elíptico, um pouco mais alongadas no sentido do comprimento máximo. O comprimento das valvas varia entre 4,5 e $10 \mathrm{~mm}$ e a altura entre 2,9 e 6,3 mm; proporção entre a altura máxima e o comprimento entre 0,62 e 0,64 . O umbo é grande e anterior. A margem dorsal é reta ou levemente arqueada. A margem ventral é arqueada. As valvas apresentam linhas de crescimen- to em número de 7 a 11, recurvadas na margem posterior próxima à margem dorsal, geralmente a partir da metade do total das linhas de crescimento. $\mathrm{O}$ espaçamento entre as linhas de crescimento é relativamente uniforme - até $0,4 \mathrm{~mm}$ na região póstero-ventral. A ornamentação está pouco preservada, porém, nos exemplares onde se pode observá-la, é pontuada. Os dados quantitativos dos espécimes encontram-se na Tabela 3.

Discussão: os exemplares são incluídos em Falsisca principalmente em vista do tamanho grande do umbo e o recurvamento das linhas de crescimento próximas à margem dorsal. A forma geral das carapaças e o tamanho do umbo também permitiriam a sua inclusão em Sinolimnadiopsis Wang (1980), porém a ornamentação é finamente pontuada e não reticulada, e o próprio gênero chinês talvez deva ser sinonimizado a favor de Falsisca (vide discussão mais adiante, no texto). Falsisca brasiliensis sp. nov. diferencia-se de outras espécies deste gênero pelo seu formato subelíptico e alongado longitudinalmente. Parece ser bem semelhante a Falsisca fabiformis Liu (1989) do Permo-Triássico da China, porém apresenta um número bem menor de linhas de crescimento e também uma margem anterior mais estreita. Falsisca brasiliensis também se assemelha a F. eotriassica eotriassica Kozur e Seidel (1982) no formato elíptico das valvas, margem dorsal reta e comprida, bandas de crescimento largas e em número médio de oito bandas, e por possuírem umbos grandes; porém, em F. brasiliensis a margem anterior tem altura nitidamente menor que a margem posterior, ocorrendo o contrário em F. eotriassica eotriassica.

\section{CONCHOSTRÁCEOS COM RECURVAMENTO DAS LINHAS DE CRESCIMENTO NA REGIÃO POSTERIOR DA VALVA}

No Brasil, foram registrados conchostráceos com recurvamento das linhas de crescimento na região posterior das valvas em depósitos do Permiano superior ao Cretáceo superior (Reed, 1929; Beurlen, 1954; Mendes, 1954; Cardoso,

Tabela 3. Dados morfométricos de Falsisca brasiliensis sp. nov.

\begin{tabular}{ccccc}
\hline Exemplar & Altura $(\mathbf{m m})$ & Comprimento $(\mathbf{m m})$ & H/L & Linhas de crescimento \\
\hline URC AC 161 Gb & 3,0 & 4,5 & 0,62 & 7 \\
DNPM 1420 C & 5,3 & 8,6 & 0,62 & 6 \\
URC AC 161 D & 2,9 & 4,7 & 0,63 & 7 \\
GP1T 1504 & 5,0 & 7,9 & 0,63 & 9 \\
DNPM 1420 B & 6,3 & 10,0 & 0,63 & 8 \\
GP1T 1509 A & 5,0 & 7,7 & 0,64 & 11 \\
\hline
\end{tabular}


1962, 1966; Rohn, 1987). Aqueles do Permiano correspondem aos exemplares da Formação Rio do Rasto (Bacia do Paraná) analisados por Reed (1929), Mendes (1954), Rohn (1987) e no presente trabalho. Conforme a revisão aqui apresentada e o estudo de novos exemplares, as espécies da Formação Rio do Rasto correspondem a $P$. subalata (Reed) e $P$. riorastensis sp. nov. da Família Palaeolimnadiopseidae e Falsisca brasiliensis sp. nov. da Família Perilimnadiidae.

O gênero Palaeolimnadiopsis foi criado por Raymond (1946) para acomodar conchostráceos fósseis com linhas de crescimento largamente espaçadas e recurvadas na porção posterior próxima à margem dorsal, semelhantes às formas viventes do gênero Limnadiopsis, mas com margem dorsal lisa, não serreada. O gênero já foi atribuído a diversas famílias, porém a Família Palaeolimnadiopseidae tem sido uma das mais usadas na literatura.

Com o avanço das pesquisas, após a introdução de Palaeolimnadiopsis, vários outros gêneros de conchostráceos fósseis, também com recurvamento das linhas de crescimento, foram sendo reconhecidos. Alguns gêneros poderiam ser sinonimizados a favor de Palaeolimnadiopsis, porém outros têm características realmente distintas quanto à forma da carapaça, ao tamanho e à posição do umbo, à presença de espinhos ou nódulos nas linhas de crescimento ou no umbo, ao número e ao espaçamento das linhas de crescimento, e à ornamentação. Tais características nortearam a proposição também de categorias taxonômicas supragenéricas, como novas famílias, ainda apresentando recurvamento das linhas de crescimento. Exemplos destes gêneros, os quais aparentemente não pertencem à Família Palaeolimnadiopseidae, são Rostroleaia, Limnadiopsileaia e Pteroleaia (Família Rostroleaiidae Novojilov, 1956); Liograpta (Família Fushonograptidae Wang, 1974); Vertexia, Tripemphigus e Pemphilimnadiopsis (Família Lioestheriidae Raymond, 1946); Ipsilonia (Família Ipsiloniidae Novojilov, 1958), Keratestheria (Família Nestoriidae) e Falsisca (Família Perilimnadiidae Chang e Chen, 1975).

Shen (1985) observou que alguns conchostráceos possuem características morfológicas que poderiam ser atribuídas a mais de uma família. Por exemplo, o gênero Pemphilimnadiopsis compartilha características das famílias Paleolimnadiidae, Vertexiidae e Paleolimnadiopseidae, devido à presença, respectivamente, de umbo grande, de nódulos no umbo e de recurvamento das linhas de crescimento na região póstero-dorsal. Este fato mostra a necessidade de se estabelecer critérios para definir qual caráter morfológico possui maior valor em diferentes níveis taxonômicos.

É interessante notar que na maioria das espécies, inclusive naquelas da Formação Rio do Rasto, não ocorre recurvamento nas primeiras linhas de crescimento, porém apenas nas últimas. Desta forma, assembléias fossilíferas compostas apenas por indivíduos jovens poderiam ser classificadas, de maneira equívoca, como pertencentes a famílias completamente distintas, sem recurvamento das linhas de crescimento.

Na última revisão publicada sobre a Família Palaeolimnadiopseidae, realizada por Shen (1985), foi mantida como principal característica diagnóstica o recurvamento das linhas de crescimento próximo à margem dorsal. De acordo com a ornamentação nas bandas de crescimento, o tamanho do umbo e a presença ou ausência de espinhos ou nódulos nas linhas de crescimento, Shen (1985) subdividiu esta família em 5 subfamílias, contendo 18 gêneros. Todas as subfamílias, exceto Sinolimnadiopseinae, apresentariam umbo pequeno.

No caso conchostráceos da Formação Rio do Rasto com linhas de crescimento recurvadas, todos haviam sido classificados inicialmente como pertencentes a Palaeolimnadiopsis, sem considerar o tamanho do umbo. Por outro lado, variações nas características relativas ao tamanho, posição e forma do umbo implicam em diferenças significativas na articulação das valvas e no desenvolvimento dos náuplios. Portanto, o caráter do umbo deve ser mais diagnóstico para a distinção de táxons do que, por exemplo, o número absoluto, a largura e a ornamentação nas bandas de crescimento, que podem variar mesmo dentro de uma mesma população (Rohn, 1986). Outro fato importante é que o tamanho do umbo implica no tamanho dos conchostráceos em seu primeiro estágio de vida antes da primeira ecdise, de modo que umbos de tamanhos muito distintos devem representar significativas diferenças na sua morfologia interna. Desta forma, justifica-se a classificação dos exemplares da Formação Rio do Rasto em Palaeolimnadiopsis Raymond, 1946 (umbo pequeno) e Falsisca Novojilov, 1970 (umbo grande). O tamanho do umbo também justifica a inclusão de cada gênero numa família distinta, respectivamente Palaeolimnadiopsidae e Perilimnadiidae.

No caso de Falsisca, cabe ser destacada a sua semelhança a Sinolimnadiopsis Wang, 1980 da China, de idade permiana. Trata-se do único gênero da Subfamília Sinolimnadiopseinae Shen, 1985, a qual pertenceria à Família Palaeolimnadopseidae na revisão de Shen (1985). Falsisca brasiliensis sp. nov. distingue-se de Sinolimnadiopsis pela ornamentação pontuada e não reticulada, porém tal fato não constitui a única justificativa para a inclusão dos exemplares estudados em Falsisca. O gênero Falsisca foi registrado em número grande de localidades da Europa e Ásia e possui mais de dez espécies descritas, com ornamentações pontuadas ou poligonais (Kozur e Seidel, 1983; Kozur, 1993; Liu, 1990). Por outro lado, Sinolimnadiopsis tem distribuição muito mais 
restrita (em poucas localidades da China) e seria o único gênero de Palaeolimnadiopseidae com umbo grande. Sugere-se que as diferenças de ornamentação não justificam a manutenção de Sinolimnadiopsis como gênero válido e, de acordo com a discussão acima, é mais provável e prático que Palaeolimnadiopseidae inclua somente táxons com umbo pequeno.

Falsisca é um gênero tipicamente permo-triássico, sendo encontrado em bacias sedimentares da Europa, da China e da Rússia (Novojilov, 1970; Kozur e Seidel, 1983; Liu, 1990), o que coaduna com as interpretações cronoestratigráficas dos estratos sedimentares da parte mais alta da Formação Rio do Rasto. Através do presente trabalho, a distribuição de Falsisca chega, portanto, ao Gondwana Ocidental, reforçando hipóteses anteriores sobre conexões continentais entre este supercontinente, a Euramérica e Cataísia, durante o Permiano (Ferreira-Oliveira e Rohn, no prelo).

Exemplares atribuídos ao gênero Palaeolimnadiopsis e à família Paleolimnadiopseidae são encontrados desde o Devoniano inferior (na Bélgica) e Carbonífero inferior (na Rússia e na França) até o Cretáceo superior (na China) (Shen, 1985; Gallego, 2005). Na América do Sul, são encontrados abundantes exemplares desta família em depósitos sedimentares do Paleozóico superior e do Mesozóico.

Considerando-se a grande amplitude estratigráfica de Palaeolimnadiopsis, inclusive em várias bacias sedimentares do mundo, o gênero não tem grande importância cronoestratigráfica. Algumas espécies do gênero podem ser bons fósseis-guias regionais, porém $P$. subalata e $P$. riorastensis restringem-se, até o momento, à parte superior da Formação Rio do Rasto e, portanto, não permitem realizar correlações com depósitos de outras bacias.

\section{CONCLUSÕES}

A reanálise dos materiais fósseis inicialmente atribuídos ao gênero $P$. subalata permitiu constatar que, na verdade, os conchostráceos com recurvamento das linhas de crescimento na região posterior da valva, encontrados em afloramentos da Formação Rio do Rasto, pertencem a três espécies diferentes, aqui atribuídas a dois gêneros: Palaeolimnadiopsis e Falsisca. Conforme observado por Shen (1985), é realmente necessária uma reavaliação dos critérios de classificação da Superfamília Palaeolimnadiopseoidea, estabelecendo-se uma ordem de caracteres das valvas que devem ser prioritários na classificação.

A presença destes dois gêneros em sedimentos da porção superior da Formação Rio do Rasto coaduna com a idade neopermiana interpretada para estes estratos (Figura 8).

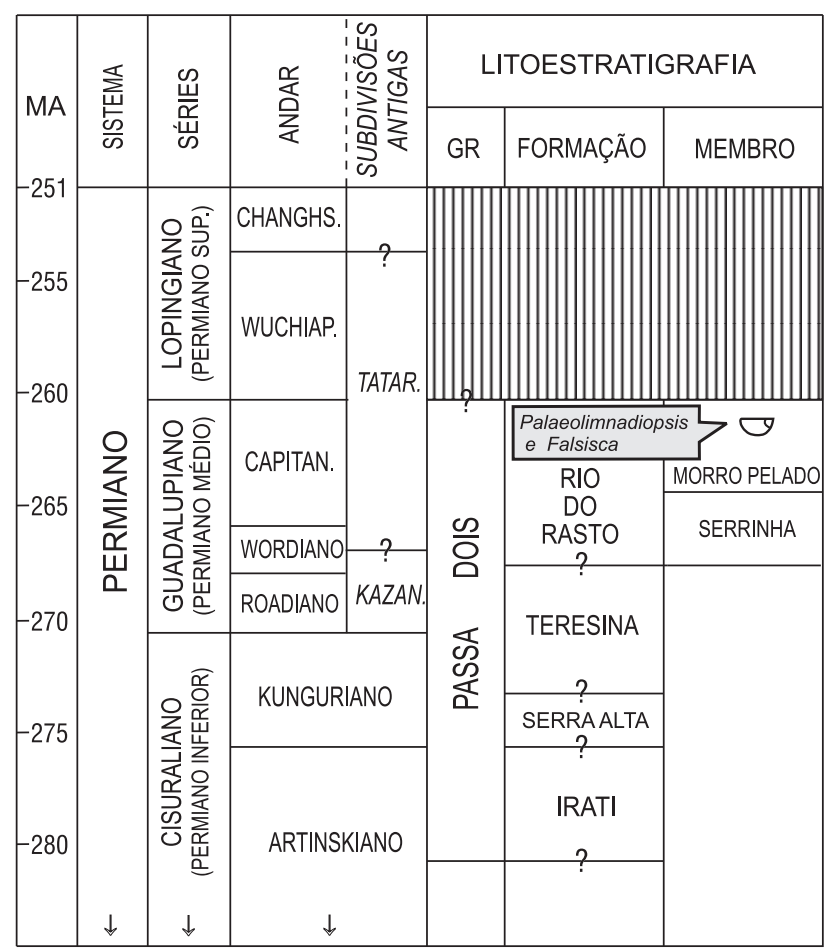

Figura 8. Litoestratigrafia e cronoestratigrafia do Grupo Passa Dois.

\section{AGRADECIMENTOS}

Agradecemos ao Ms.Thiago Meglhioratti (Petrobrás) e ao Luis Fernando M. Montano (Curso de Geologia - UNESP/ Rio Claro) pela colaboração nos trabalhos de campo, ao Dr. Thomas R. Fairchild (IGc/USP) e à Dra Rita de Cássia Cassab (DNPM) por terem facilitado o acesso aos exemplares das coleções respectivamente do Instituto de Geociências da Universidade de São Paulo e do Departamento Nacional de Produção Mineral (DNPM). Agradecemos também ao Prof. Dr. Elliot Kitajima e ao Núcleo de Apoio à Pesquisa em Microscopia Eletrônica Aplicada a Agricultura (NAPMEPA), onde foram feitas as imagens no MEV. A pesquisa foi financiada, em parte, pelo CNPq, PRH-05 (ANP) e FAPESP(05/55027-4).

\section{REFERÊNCIAS BIBLIOGRÁFICAS}

CARDOSO, R. N. Alguns conchostráceos mesozóicos do Brasil. Boletim da Sociedade Brasileira. de Geologia, v. 11, n. 2, p. 21-32, 1962.

CARDOSO, R. N. Conchostráceos do Grupo Bahia: Brasil. Boletim do Instituto de Geologia, Escola Federal de Minas de Ouro Preto, v. 1, n. 2, p. 43-76, 1966. 
CHEN, Pei-ji; SHEN, Yan-bin. Fossil conchostraca. Beijing: Science Press, 1985. p. 241 (In Chinese).

FERREIRA-OLIVEIRA, L. G; ROHN, R. Leaiid conchostracans from the uppermost permian strata (Capitanian) of the Paraná Basin, Brazil: chronostratigraphic and paleobiogeographic implications. Journal of South American Earth Sciences. (no prelo).

GALLEGO, O. F. First record of the family palaeolimnadiopseidae Defretin-LeFranc, 1965 (CrustaceaConchostraca) in the Triassic of Argentina. Journal of South American Earth Science, v. 18, n. 2, p. 223-231, 2005.

KOZUR, H. W. Range charts of conchostracans in the Germanic Buntsandstein. In: LUCAS, S.G.; MORALES, M. (Ed.) The nonmarine triassic. New Mexico: Museum of Natural History e Science, 1993. 1993. p. 249-253. (Bulletin, 3).

KOZUR, H. W.; SEIDEL, G. Revision der ConchostracenFaunendes unteren und mittleren Buntsandsteins. Teil I. Zeitschrift fur Geologische Wissenschaften, v. 11, p. 295-423, 1983.

LIU, S. Conchostracans in baicheng district of Xinjiang. Xinjiang Geology, v. 8, n. 2, p. 176-184, 1990.

MENDES, J. C. Conchostráceos permianos do Brasil. In: LANGE, F.W. (ed.) Paleontologia do Paraná. Curitiba: Museu Paranaense, 1954. p.153-164. est.12-14. (Volume Comemorativo do $1^{\circ}$ Centenário do Estado do Paraná ).

NOVOJILOV, N. I. Superfamily limnadioidea (Conchostraca). Moskva: Izdat “Nauka”, 1970. 237 p.

RAYMOND, P. E. The genera of fossil Conchostraca an order of bivalved Crustacea. Bulletin of the Museum of Comparative Zoology at Harvard, v. 96, n. 3, p. 217-307, 1946.

ROHN, R. Caracteres diagnósticos dos conchostráceos fósseis - exemplos da Formação Rio do Rasto (Permiano Superior, Bacia do Paraná). Anais da Academia Brasileira de Ciências, Rio de Janeiro, v. 58, n. 4, p. 596-596, 1986.

ROHN, R. Conchostráceos da Formação Rio do Rasto (Bacia do Paraná, Permiano Superior) no Estado do Paraná e no norte do Estado de Santa Catarina. Boletim do Instituto de Geociências, Universidade de São Paulo, Série Cientifica, v. 18. p. 27-66, 1987.

ROHN, R. Evolução ambiental da Bacia do Paraná du- rante o neopermiano no leste de Santa Catarina e do Paraná. 1994. 250 f. Tese (doutorado) - Instituto de Geociências, Universidade de São Paulo, São Paulo, 1994.

REED, F. R. C. Novos phyllopodos fósseis do Brasil. Boletim do Serviço Geológico e Mineralógico do Brasil, Rio de Janeiro, v. 34, n. 17, p. 2-16, 1929.

SHEN, Yan-bin. Classification and evolution of family palaeolimnadiopseidae (Conchostraca). Scientia Sinica, Series B, v. 28, n. 8, p. 888-894, 1985.

TASCH, P. Fossil Conchostraca of the southern hemisphere and continental drift; paleontology, biostratigraphy and dispersal. Geological Society of America Memoir, n. 165, p. 1-290, 1987. 